\title{
醏
}

Máximo Santos Miranda*

\section{LA CRISIS DE LA BANCA EUROPEA El caso particular del sistema bancario de Montenegro}

Montenegro es un pequeño país europeo, con una población de poco más de 600.000 habitantes, que fue reconocido como país independiente en el año 2006 por parte de la comunidad internacional y que aspira a formar parte de la Unión Europea en el futuro cercano. En este contexto, el presente trabajo analiza cómo se ha comportado su sector bancario en un entorno de fuerte crisis financiera internacional, que sobrevino después de un periodo muy traumático, política y económicamente, por la crisis en Yugoslavia. Y todo ello teniendo en cuenta que el país no cuenta con una moneda propia, ya que tiene al euro como moneda oficial, y con la peculiaridad de que Montenegro no forma parte del Eurosistema ni es aún miembro de la Unión Europea.

Palabras clave: Montenegro, crisis, sistema financiero, flujos monetarios.

Clasificación JEL: E50, E58, G21.

\section{Introducción}

Montenegro formó parte de la República Federal Socialista de Yugoslavia, Estado que estaba conformado por seis repúblicas ${ }^{1}$ y dos regiones autónomas ${ }^{2}$, y que comenzó a desintegrarse a partir del año 1991 a consecuencia de las sucesivas guerras yugoslavas. El germen más reciente de la desmembración del país, sin remontarnos a otras etapas históricas anteriores, hay que situarlo en el año $1980^{3}$ cuando el país vivió una profunda crisis económica, que hizo aumentar las tensiones entre los

\footnotetext{
* Departamento de Economía Aplicada de la Universidad Nacional de Educación a Distancia.

Versión de mayo de 2015.

1 Las seis repúblicas que lo conformaban eran la República Socialista de Bosnia y Herzegovina, la República Socialista de Croacia, la República Socialista de Eslovenia, la República Socialista de Macedonia, la República Socialista de Montenegro y la República Socialista de Serbia.

2 Vojvodina y Kosovol.

3 Tras la muerte de Josip Broz Tito que dirigió al país desde el final de la Segunda Guerra Mundial.
}

diferentes pueblos que conformaban la Federación Yugoslava.

Tras el ascenso de partidos nacionalistas al poder en Serbia, dos de sus repúblicas constituyentes (Eslovenia y Croacia) declararon su independencia en 1991, a las que seguirían Macedonia y Bosnia-Herzegovina al año siguiente, no sin resistencia por parte de Serbia. De esta forma, en 1991 la tensión entre las diferentes repúblicas derivó en un sangriento conflicto conocido como las guerras de Yugoslavia, que hicieron que la muerte y la destrucción volvieran al continente europeo después de décadas de paz, aunque este periodo de estabilidad siempre estuviese sometido a las tensiones propias de la guerra fría. Estas declaraciones de independencia redujeron paulatinamente el tamaño de Yugoslavia, pasándose a ser denominado, a partir de 2003, Serbia y Montenegro.

Montenegro fue la última república que se separó de la Federación Yugoslava, convirtiéndose $\triangleright$ 
en Estado independiente después del referéndum que tuvo lugar el 21 de mayo del $2006^{4}$. Tras su celebración, el reconocimiento de Montenegro como Estado independiente fue inmediato por parte de la comunidad internacional ${ }^{5}$. Posteriormente, Montenegro promulgó su nueva Constitución ${ }^{6}$, lo que implicó, entre otras cosas, el primer paso en el proceso de adhesión ${ }^{7}$ a la Unión Europea, iniciándose desde ese momento las negociaciones para integrarse en la Unión. Ello supone la necesaria adecuación de su legislación al acervo comunitario y, entre otras cosas, implica la trasposición al derecho interno de todas las directivas europeas en materia de regulación y supervisión financiera.

Desde el momento en que el país se separó de Serbia, Montenegro comenzó a ser miembro de las principales organizaciones financieras internacionales tales como el Banco Europeo de Reconstrucción y Desarrollo, el Banco Mundial, el Fondo Monetario Internacional o la Organización Mundial del Comer$\mathrm{cio}^{8}$. Estas adhesiones han tenido un impacto muy positivo en Montenegro, no sólo en lo referente a las relaciones comerciales con terceros países, sino que además ha mejorado la instauración de un clima favorecedor a la inversión extranjera9 ${ }^{9}$.

Finalmente resaltar en esta introducción que Yugoslavia, a pesar de ser un país socialista, eligió

\footnotetext{
4 El resultado del referéndum fue que el 55 por 100 de los votantes optaron por la independencia de Serbia. La Declaración de Independencia fue refrendada por el Parlamento de Montenegro el 3 de junio del año 2006.

5 A este reconocimiento internacional ayudó de forma muy importante el reconocimiento de los resultados por parte de los cinco miembros permanentes del Comité de Seguridad de las Naciones Unidas el 23 de mayo del año 2006.

6 En el mes de octubre del año 2007.

7 Montenegro oficialmente solicitó ser miembro de la Unión Europea en el mes de diciembre de 2008, siendo designado como candidato a la integración en el mes de diciembre del 2010. En junio del año 2012 el Consejo de la Unión decidió recomendar negociaciones de adhesión de Montenegro a la Unión.

8 La incorporación a dicho organismo se produjo en el mes de abril del año 2012.

9 Las compañías nacionales y extranjeras reciben el mismo tratamiento legal. Los inversores extranjeros están autorizados a invertir en cualquier tipo de industria y a transferir libremente los beneficios obtenidos a los países de origen de la inversión. Sólo hay dos restricciones a la inversión extranjera: 1) No se puede invertir en la industria armamentística (sí se puede invertir en este sector como accionista minoritario de una empresa fabricante de armas, siempre que el accionista mayoritario sea montenegrino). 2) No se pueden adquirir recursos naturales, bienes históricos o propiedades de marcado interés público.
}

un funcionamiento diferente al de la URSS y al del resto de sus países satélites de la Europa oriental, no siendo miembro de ninguna de las alianzas militares dominantes en Europa (OTAN y Pacto de Varsovia) ${ }^{10}$. De esta forma, el país funcionó, desde el final de la Segunda Guerra Mundial hasta los años ochenta, con un sistema socioeconómico denominado socialismo autogestionario. Sistema que fue percibido en muchos casos como una tercera vía al modelo económico capitalista y al impulsado por la URSS.

\section{Situación de la economía de Montenegro}

La economía de Montenegro atravesó por un periodo muy complicado desde que se desataron las guerras yugoslavas hasta mediados de la primera década del siglo xxI, ya que su economía estuvo muy unida a la de Serbia hasta la independencia del país. Montenegro al igual que Serbia estuvo sometido a sanciones económicas ${ }^{11}$, que debilitaron aún más su maltrecha economía. La situación económica se debilitó tanto que el Banco Central de Montenegro estimó que en 1998, dos tercios de la población del país se encontraba por debajo del umbral de la pobreza. La situación económica en esos años fue tan compleja que el país alcanzó una tasa de inflación de tres millones por 100 en enero de 1994. Debido a esta difícil situación económica, con la paralización de la práctica totalidad de la industria montenegrina, como consecuencia de las sanciones y del desmembramiento del país, y a su estratégica situación geográfica ${ }^{12}$, Montenegro se convirtió en un $\triangleright$

\footnotetext{
10 Yugoslavia fue uno de los países impulsores del Movimiento de los Países No Alineados desde 1956.

11 La ONU impuso en el mes de mayo del 2012 fuertes sanciones económicas a Montenegro a consecuencia de las guerras yugoslavas. Este hecho y la desintegración del mercado yugoslavo propiciaron la mayor crisis por la que atravesó el país desde el final de la Segunda Guerra Mundial. Las pérdidas ocasionadas por las sanciones internacionales fueron estimadas por el Banco Central de Montenegro en 6.390 millones de dólares, cifra muy relevante para una economía en que el PIB del año 2013 fue de 4.416 millones de dólares.

12 Montenegro tiene acceso al mar Adriático y acceso marítimo a Albania a través del lago Skadar.
} 
centro internacional de contrabando de bienes, como el combustible o el tabaco. Estas prácticas se mantuvieron durante al menos un decenio, convirtiéndose de facto en una práctica legalizada.

En el periodo en el que el país estuvo unido exclusivamente con Serbia, Montenegro tuvo su propio Banco Central y lo que es más determinante, adoptó primero como moneda oficial el marco alemán, con el objeto de luchar contra las altas tasas de inflación que tuvo su economía y, a partir del año 2002, el euro en detrimento del dinar yugoslavo.

Montenegro experimentó, entre 2006 y 2008 , una vez que el conflicto yugoslavo y las sanciones internacionales habían finalizado, un fortísimo crecimiento económico, basado fundamentalmente en una fuerte explosión inmobiliaria, en la que inversores rusos y británicos se lanzaron a adquirir propiedades apresuradamente en la costa montenegrina. A partir de ese momento, Montenegro recibió un fuerte volumen de inversión extranjera que impulsó los dos sectores claves de su crecimiento, el turismo de lujo y las infraestructuras. Las autoridades favorecieron este modelo de crecimiento, ya que su objetivo no era otro que convertir a Montenegro en un destino de turismo de lujo para los ciudadanos europeos.

\begin{tabular}{|l|c|c|c|} 
TABLA 1 \\
CRECIMIENTO DE LA ECONOMÍA DE MONTENEGRO \\
ENTRE LOS AÑOS 2006 Y 2009 \\
(En porcentaje) \\
\hline Año & Crecimiento del PIB & Año & Crecimiento del PIB \\
\hline 2006 & 8,6 & 2008 & 6,9 \\
\hline 2007 & 10,7 & 2009 & $-5,7$ \\
\hline Fuente: Banco Mundial. & & \\
\hline
\end{tabular}

Tras el desmembramiento de la República Federal de Yugoslavia, la industria de Montenegro se vio abocada a un continuo declive, siendo sustituida paulatinamente por el mayor peso del sector servicios. Es el sector servicios el gran generador de empleo en el país, ocupando al 76 por $100^{13}$ de la población activa. En la actualidad podemos definir a la economía de Montenegro como una economía de servicios ${ }^{14}$, en la que el sector

\footnotetext{
13 Datos del año 2014.

14 El sector servicios representa el 73,26 por 100 del PIB, la industria el 16,66 por 100 y el sector agrícola el 10,08 por 100 según datos del año 2012.
}

turístico ocupa un lugar muy destacado y que se encuentra todavía en un estado de transición entre la economía socialista anterior y la economía de mercado.

\begin{tabular}{|c|c|c|c|}
\hline \multicolumn{4}{|c|}{$\begin{array}{l}\text { TABLA } 2 \\
\text { CRECIMIENTO DE LA ECONOMÍA DE MONTENEGRO } \\
\text { ENTRE LOS AÑOS } 2010 \text { Y } 2015 \\
\text { (En porcentaje) }\end{array}$} \\
\hline Año & Crecimiento del PIB & Año & Crecimiento del PIB \\
\hline 2010 & 0,50 & 2013 & 3,30 \\
\hline 2011 & 3,20 & 2014 & 1,50 \\
\hline 2012 & $-2,50$ & $2015^{*}$ & 3,40 \\
\hline
\end{tabular}

Desde su independencia, se inició por parte de las autoridades de Montenegro un vasto programa de privatizaciones que ha propiciado que en la actualidad la empresa pública, otrora absoluta dominadora de la economía montenegrina, ocupe hoy en día un papel marginal.

\begin{tabular}{|c|c|c|c|c|}
\hline \multicolumn{5}{|c|}{$\begin{array}{c}\text { TABLA } 3 \\
\text { INVERSIONES DIRECTAS EN MONTENEGRO } \\
\text { ENTRE LOS AÑOS 2011 Y } 2014 \\
\text { (En millones de USD) }\end{array}$} \\
\hline Año & 2011 & 2012 & 2013 & $2014^{\star}$ \\
\hline Inversión directa & 558 & 620 & 447 & 480 \\
\hline
\end{tabular}

La atracción de inversión extranjera al país balcánico se puede fundamentar en los siguientes elementos:

1. Las compañías extranjeras tienen los mismos derechos que las montenegrinas.

2. El tipo impositivo es uno de los más reducidos y competitivos de Europa ${ }^{15}$.

3. La mano de obra es bastante cualificada y sus salarios relativamente reducidos, no sólo en comparación con los países de la Unión Europea, sino con otros países vecinos.

4. La moneda oficial es el euro, lo que dota a su economía de estabilidad.

5. La burocracia necesaria para crear una sociedad es simple y rápida, lo que le otorga una indudable ventaja en relación a otros países limítrofes.

\footnotetext{
15 Actualmente se sitúa en el 9 por 100.
} 
6. Montenegro es un país en la actualidad democrático y estable.

Sin embargo, también hay aspectos negativos en lo que a la atracción de inversiones se refiere. Así, el país cuenta con un déficit por cuenta corriente notable, con una considerable deuda exterior, presenta problemas extendidos de corrupción ${ }^{16}$ y la regulación relativa a la propiedad intelectual es prácticamente inexistente. No obstante lo anterior, los flujos de inversión directa en el país son notables, siendo los mayores inversores en estos últimos cuatro años Suiza, Noruega, Austria, Rusia e Italia.

\begin{tabular}{|c|c|c|c|}
\hline \multicolumn{4}{|c|}{$\begin{array}{c}\text { TABLA } 4 \\
\text { BALANZA POR CUENTA CORRIENTE } \\
\text { ENTRE LOS AÑOS } 2010 \text { Y } 2015 \\
\text { (En porcentaje) }\end{array}$} \\
\hline Año & Porcentaje del PIB & Año & Porcentaje del PIB \\
\hline 2010 & $-20,60$ & 2013 & $-14,60$ \\
\hline 2011 & $-17,70$ & 2014 & $-15,70$ \\
\hline 2012 & $-18,70$ & $2015^{\star}$ & $-16,30$ \\
\hline
\end{tabular}

Respecto a otros indicadores como la inflación, esta se mantiene en niveles muy reducidos. A ello ayuda especialmente el que el país adoptase el euro como moneda oficial. La tasa de desempleo se situó en 2013 en el 19,6 por 100 sobre la población activa, lo que representa un porcentaje muy elevado.

\begin{tabular}{|c|c|c|c|c|}
\hline \multicolumn{5}{|c|}{$\begin{array}{c}\text { TABLA } 5 \\
\text { TASA DE INFLACIÓN EN MONTENEGRO } \\
\text { ENTRE LOS AÑOS } 2010 \text { Y } 2013 \\
\text { (En porcentaje) }\end{array}$} \\
\hline Año & 2010 & 2011 & 2012 & 2013 \\
\hline Tasa de inflación & 1,60 & 0,90 & $-0,10$ & 2,20 \\
\hline \multicolumn{5}{|c|}{ Fuente: Banco Mundial. } \\
\hline
\end{tabular}

\section{La crisis financiera internacional y el sistema bancario de Montenegro}

\subsection{El sistema bancario de Montenegro anterior a la independencia}

Si la economía montenegrina ha sufrido enormes cambios en las últimas décadas, también el

\footnotetext{
16 Con el objeto de combatir la corrupción, el Gobierno creó a mediados de la primera década del siglo XXI una agencia anticorrupción, encargada de preparar la legislación que la combata, mejorando la transparencia de las transacciones financieras y de las operaciones comerciales. Como consecuencia, se han conseguido ciertos logros al respecto, aunque todavía queda mucha distancia que recorrer. No obstante, la lucha contra la corrupción es uno de los asuntos claves que está impulsando el actual Gobierno.
}

sector bancario ha sufrido una enorme reconversión, como consecuencia de la transición de la economía de Montenegro desde un esquema socialista a uno de mercado. Sin embargo hay que destacar que el sistema bancario de la antigua Yugoslavia presentaba importantes diferencias con los sistemas financieros de los países del COMECON. De hecho las autoridades económicas yugoslavas, de entre los años 1960 y 1980, lo consideraban un sistema intermedio entre los sistemas bancarios de planificación central y los sistemas bancarios capitalistas. Los bancos disponían de bastante autonomía y acogieron algunas de las prácticas bancarias occidentales, aunque su operativa se viera muy limitada por la rigidez del mercado en que se desenvolvían y por la imposibilidad que tenían de obtener beneficio. Pero aun así se puede considerar que entre 1960 y 1980 el sistema bancario de Yugoslavia era el más avanzado de los países de economía planificada. Prueba de ello es que entre 1970 y 1980 ningún banco fue liquidado ni declarado en quiebra.

A partir de 1980 Yugoslavia atravesó una profunda crisis económica que afectó, como no podía ser de otro modo, a su sistema bancario. Muchos bancos tuvieron problemas en cobrar sus créditos, al mismo tiempo que no disponían de las divisas necesarias con las que hacer frente a sus obligaciones exteriores ${ }^{17}$. Los bancos y el país sufrieron una importante crisis de deuda, que finalizó con el aplazamiento de la misma tras los acuerdos de los clubes de Londres y París. Como consecuencia de todo ello, se promulgó una nueva ley bancaria que perseguía resolver los problemas de endeudamiento de los bancos. Sin embargo, la reforma no tuvo éxito a consecuencia de la desintegración yugoslava. A partir de ese momento, los bancos de Serbia y Montenegro entraron en una situación extremadamente difícil a consecuencia de las sanciones internacionales, las guerras, la paralización de la industria, la hiperinflación, la ausencia de divisas y, la morosidad del crédito. Consecuencia de ello fue que el Gobierno de Serbia-Montenegro decidiera $\triangleright$

17 Entre 1970 y 1980 el sistema bancario yugoslavo obtuvo muchos créditos del exterior, en moneda diferente del dinar yugoslavo, que fueron avalados por el Gobierno yugoslavo. La devaluación del dinar yugoslavo y la fuerte crisis económica impidieron su repago. 
liquidar sus cuatro mayores entidades bancarias ${ }^{18}$ en el 2002 ante las enormes pérdidas que presentaban y ante las negativas perspectivas esperadas en los siguientes dos ejercicios. Los ciudadanos serbios y montenegrinos dejaron de confiar en sus bancos, retirando sus depósitos de forma masiva.

La introducción del marco alemán y el importante papel supervisor e independiente que a partir de 2001 desempeñó el Banco Central de Montenegro, contribuyeron a que el país se comenzara a independizar de la influencia de Serbia e iniciara la reforma de su sistema bancario. En consecuencia, Montenegro comenzó a reformar su sistema bancario con una década de retraso respecto a lo que hicieron otros países de Europa Central y del Este.

El año 2002 se puede considerar un año clave para el sistema bancario montenegrino, reduciéndose a diez el número de bancos existentes, casi los mismos que actualmente, de los cuales sólo dos tenían a bancos extranjeros como principales accionistas. Montenegrobanka, que era la principal institución bancaria del país, fue reestructurada entre 2002 y 2003, siendo privatizado en la segunda mitad del 2003 y pasando a ser el capital extranjero el mayoritario. A finales de 2005, justo antes de la declaración de independencia del país, Montenegro contaba con diez bancos, de los cuales las tres mayores entidades ${ }^{19}$ controlaban el 66 por 100 de los activos bancarios.

\subsection{El sistema bancario montenegrino tras la Declaración de Independencia del país}

El sector bancario de Montenegro, que comenzó a estabilizarse a partir del 2003, experimentó enormes crecimientos en los años siguientes, tanto en lo referente al aumento de depósitos como de créditos. De hecho en los años previos

\footnotetext{
18 Jugobanka, Beobanka, Beogradsla e Investbanka. Estos cuatro bancos contaban con el 57 por 100 del total de los activos del sector bancario del país.

19 El mayor banco del país era el privado Crnogorska Komercialna Banka.
}

\begin{tabular}{|c|c|c|c|}
\hline \multicolumn{4}{|c|}{$\begin{array}{c}\text { TABLA } 6 \\
\text { PARTICIPACIÓN EXTRANJERA DE LOS MAYORES BANCOS } \\
\text { DE LAS EXREPÚBLICAS YUGOSLAVAS EN EL AÑO } 2005\end{array}$} \\
\hline República & $\begin{array}{c}\mathrm{N}^{\circ} \text { de } \\
\text { sucursales }\end{array}$ & Mayor banco & $\begin{array}{l}\text { Participa. } \\
\text { extranjera } \\
\quad(\%)\end{array}$ \\
\hline Bosnia y Herzegovina & 33 & Raiffeisen Banka & 70,20 \\
\hline Croacia & 34 & Zagrebacka Banka & 91,30 \\
\hline Macedonia & 20 & $\begin{array}{l}\text { Komercijalna Banka ad } \\
\text { Skoplje }\end{array}$ & 49,00 \\
\hline Montenegro & 10 & $\begin{array}{l}\text { Crnogorska Komercijalna } \\
\text { Banka }\end{array}$ & 68,00 \\
\hline Serbia & 40 & Raiffeisen ad Beograd & 67,00 \\
\hline Eslovenia & 19 & NLB & 35,10 \\
\hline
\end{tabular}

cas yugoslavas.

TABLA 7

MAYORES BANCOS DE MONTENEGRO POR VOLUMEN DE ACTIVOS EN EL AÑO 2012

\begin{tabular}{|l|l|c|}
\hline Nombre del banco & \multicolumn{1}{|c|}{ Propietario } & \multicolumn{1}{|c|}{$\begin{array}{c}\text { Activos } \\
\text { (Millones de euros) }\end{array}$} \\
\hline CKB & $\begin{array}{l}\text { OTP Group } \\
\text { (Hungría) }\end{array}$ & 840 \\
\hline $\begin{array}{l}\text { NLB } \\
\text { Montenegrobanka }\end{array}$ & $\begin{array}{l}\text { NLB Group } \\
\text { (Eslovenia) }\end{array}$ & 520 \\
\hline $\begin{array}{l}\text { Hypo Alpe-Adria Bank } \\
\text { AD }\end{array}$ & $\begin{array}{l}\text { Hypo Group Alpe } \\
\text { Adria (Austria) }\end{array}$ & 507 \\
\hline $\begin{array}{l}\text { PRBA Banka Crne } \\
\text { Gore }\end{array}$ & $\begin{array}{l}\text { Private Company } \\
\text { (Montenegro) }\end{array}$ & 367 \\
\hline $\begin{array}{l}\text { Podgoricka Banka } \\
\text { Societé Generale } \\
\text { (Francia) }\end{array}$ & 240 \\
\hline Fuente: elaboración propia. Asociación Bancaria de Montenegro.
\end{tabular}

al estallido de la crisis, el sistema bancario montenegrino registraba espectaculares aumentos en el volumen de depósitos y créditos. Aumentos que estaban muy por encima de los de otros países europeos.

Unos incrementos que en los años 2006 y 2007 fueron incluso superiores al 90 por 100 y que se pueden explicar fundamentalmente por dos elementos:

1. La crisis económica y la política anterior desbancarizaron casi completamente la economía montenegrina, hasta el punto que gran parte de la población realizaba toda su actividad económica en efectivo, sin utilizar en ningún caso a los intermediarios financieros. Cabe recordar en este punto que en los peores años de la crisis, gran parte de la economía era sumergida, con un gran peso del contrabando.

2. A partir del 2004 se instaura una estabilidad política no conocida en los últimos años. El $\square$ 
apoyo de la Unión Europea y el deseo de las autoridades en la adhesión del país a la Unión, la integración del país en diversas organizaciones internacionales y el uso del euro como moneda oficial en detrimento del dinar yugoslavo apoyaron a esta estabilidad.

\begin{tabular}{|l|r|r|r|r|r|}
\hline \multicolumn{7}{c|}{$\begin{array}{c}\text { TABLA 8 } \\
\text { CRECIMIENTO DE LOS DEPÓSITOS BANCARIOS ENTRE LOS } \\
\text { AÑOS 2005 Y 2009 } \\
\text { (En porcentaje) }\end{array}$} \\
\hline \multicolumn{1}{|c|}{ Año } & 2005 & 2006 & 2007 & 2008 & 2009 \\
\hline Incremento & 78,60 & 120,50 & 94,40 & $-4,80$ & $-8,30$ \\
\hline Fuente: Banco Central de Montenegro. & & \\
\hline
\end{tabular}

Pero no sólo aumentaron enormemente los depósitos en esos años sino que también lo hicieron los créditos. Un crecimiento del crédito que fue incluso superior al de los depósitos entre los años 2006 y 2008. A este hecho ayudó de forma importante el boom inmobiliario experimentado entre 2005 y 2008 en lo que al sector turístico de la costa adriática se refiere y a que el euro fuera la moneda de curso legal. Los crecimientos del crédito fueron tan intensos que, en otoño del 2007. el Banco Central se vio obligado a tomar diversas medidas con el objeto de reducir su crecimiento.

\begin{tabular}{|l|c|c|c|c|c|c|}
\multicolumn{7}{c|}{$\begin{array}{l}\text { TABLA } 9 \\
\text { CRECIMIENTO DEL CRÉDITO BANCARIO ENTRE } \\
\text { LOS ANNOS 2005 Y } 2010 \\
\text { (En porcentaje) }\end{array}$} \\
\hline Año & 2005 & 2006 & 2007 & 2008 & 2009 & 2010 \\
\hline Incremento & 33,60 & 125,30 & 165,10 & 24,60 & $-14,30$ & $-11,60$ \\
\hline
\end{tabular}

El sistema bancario de Montenegro contaba a 31 de diciembre del 2012 con 2.325 empleados, a los que hay que añadir los 334 empleados $^{20} \mathrm{del}$ Banco Central. Y todo ello en un país en el que operaban un total de once entidades y seis pequeñas instituciones financieras especializadas en microcréditos.

\footnotetext{
20 De ellos 44 empleados trabajan en la labor de supervisión del sistema bancario del país.
}

\begin{tabular}{|c|c|c|}
\hline \multicolumn{3}{|c|}{$\begin{array}{c}\text { TABLA } 10 \\
\text { MAYORES BANCOS DE MONTENEGRO } \\
\text { POR NÚMERO DE OFICINAS EN EL AÑO } 2012\end{array}$} \\
\hline Nombre del banco & $\begin{array}{l}\text { Número } \\
\text { de oficinas }\end{array}$ & $\begin{array}{c}\text { Año } \\
\text { de su fundación }\end{array}$ \\
\hline Atlas Banka Montenegro & 22 & 2002 \\
\hline CKB & 30 & 1997 \\
\hline Hypotekarna Banka & 16 & nd \\
\hline NLB Montenegro Banka & 18 & 1905 \\
\hline Pvra Banka Crn Gore & 35 & 1901 \\
\hline Erste Bank Montenegro & 17 & $2009(1)$ \\
\hline Societe Generale Montenegro & 20 & nd \\
\hline \multicolumn{3}{|c|}{$\begin{array}{l}\text { (1) El banco austriaco entra en Montenegro tras la compra del } 100 \\
\text { por } 100 \text { del capital del banco Montenegrino Opportunity Bank Podorica. } \\
\text { Nd: no disponible. } \\
\text { Fuente: elaboración propia. Banco Central de Montenegro. The } \\
\text { Banker. }\end{array}$} \\
\hline
\end{tabular}

Actualmente no se permite que se establezcan sucursales bancarias de entidades de terceros países, sino que siempre es necesario que la banca extranjera se establezca a través de filiales locales sujetas enteramente a la legislación montenegrina.

Fruto de la necesidad de modernización de su regulación bancaria y del deseo por parte de las autoridades montenegrinas de que el país se adhiera a la Unión Europea, Montenegro ha emprendido un largo proceso de trasposición a su legislación de diversas directivas comunitarias relativas a las entidades y servicios financieros. En este sentido Montenegro ha traspuesto en gran medida la Directiva 2006/48/EC, que regula las condiciones para el establecimiento y autorización a operar en el país de las entidades de crédito, así como lo relativo a la supervisión de dichas entidades.

La nueva ley bancaria dictamina que las instituciones de crédito que quieran operar en Montenegro necesitan una autorización de su banco central para establecerse y operar en el país, proveyendo la nueva ley una exhaustiva lista de condiciones que garantizan o impiden la concesión o revocación de la licencia bancaria en Montenegro. Los elementos de la Directiva 2006/48/EC relativos a las mejores prácticas bancarias acerca del manejo de la liquidez, a la gestión del riesgo, al manejo de las auditorías internas o de la aplicación de los procesos de supervisión de entidades bancarias han sido íntegramente desarrolladas por la nueva ley bancaria. Ello no obsta, para que todavía existan algunos elementos de la $D$ 
citada directiva que no han sido desarrollados por la normativa interna del país pero a los que sus autoridades se han comprometido a transponer en los próximos meses.

Igualmente, la ley bancaria también ha traspuesto al derecho montenegrino la Directiva 2006/49/EC en lo relativo a la adecuación del capital de entidades financieras y empresas de inversión. En este sentido hay que destacar que entre 2007 y 2013 el índice de solvencia de los bancos montenegrinos fue constantemente superior al mínimo señalado por la regulación europea. El Banco Central de Montenegro tiene la potestad de obligar a los bancos del país a someterse a distintas pruebas de liquidez utilizando para las mismas distintos supuestos de partida que implican niveles severos de dificultad en la economía de Montenegro. En función de los resultados obtenidos en dichas pruebas, los bancos podrán ser obligados a recapitalizarse adecuadamente $o$ a adoptar distintos sistemas de contingencia con el objeto de evitar posibles dificultades futuras. Otras directivas comunitarias como la 2010/76/EU o la 2009/111/ EC todavía no se encontraban traspuestas en su ley bancaria. La Directiva 2002/87/EC relativa a la estructura de supervisión de los conglomerados financieros tampoco ha sido traspuesta, aunque sus autoridades se han comprometido a hacerlo para comienzos del año 2018.

Finalmente destacar que Montenegro adoptará una nueva ley relativa a instituciones de crédito a finales del año 2015 , con el objeto de adaptar a su sistema bancario a los requerimientos de Basilea III. Dicha ley entrará en vigor el 1 de enero del año 2017.

\subsection{El sistema bancario de Montenegro y la crisis financiera internacional}

La crisis financiera internacional afectó profundamente al sector bancario montenegrino como consecuencia del gran endeudamiento de su economía con el exterior, a sus déficits comerciales y a la escasez de reservas de divisas del país. Los años previos al inicio de la crisis se caracterizaron por ser años de crecimientos verticales en lo que a la concesión de crédito se refiere, sin que en muchos casos se midiera adecuadamente el nivel de riesgo de los mismos. Entre 2005 y 2008 la entrada masiva de capitales, junto con el aumento de la competencia bancaria y el mayor apetito por el riesgo dispararon la concesión de créditos, en un país que después de varias décadas comenzaba a resurgir económicamente con muchísima fuerza.

Su sistema financiero no sufrió la primera oleada de la crisis al no tener sus entidades bancarias productos estructurados que vieron caer súbitamente su valor, como sí ocurrió en otros sistemas bancarios como el británico, alemán, holandés o norteamericano. Su sistema financiero se había concentrado fundamentalmente, en los años previos a la crisis, en la concesión de créditos hipotecarios, y no en otro tipo de inversiones más complejas. Sin embargo, el sistema bancario montenegrino si se vio afectado por la segunda ola de la crisis financiera. Como resultado de la misma, las divisas dejaron de llegar de forma tan masiva, como sucedió en los años previos, y al mismo tiempo las exportaciones del país cayeron de forma notable, siendo el turismo ${ }^{21}$, principal fuente de recursos de Montenegro, el que mantuvo a flote su economía, que no obstante decreció un 5,7 por 100 en el año 2009.

Como consecuencia de todo lo anterior, en el año 2009, Montenegro sufrió una enorme contracción del crédito, al mismo tiempo que el valor de los activos, que estaban en posesión de las entidades financieras, comenzaron a deteriorarse de manera significativa. Esta contracción repentina del crédito, como no podía ser de otro modo, afectó de forma notable a la economía real en el año 2009 , de forma que algunas entidades bancarias incluso suspendieron totalmente la concesión de nuevos créditos durante algunos meses entre los años 2009 y 2010. Sin embargo, hay que destacar que pocas entidades bancarias $\triangle$

21 Entre las medidas anticíclicas tomadas por el Gobierno montenegrino destacó la fuerte inversión publicitaria en el sector turístico del país a nivel internacional. 
tuvieron problemas severos de liquidez y que las que los tuvieron fueron fuertemente apoyadas por sus entidades matrices extranjeras. Este apoyo se materializó en la recapitalización de sus filiales con el objeto de reforzar su solvencia.

Como consecuencia, el Gobierno se vio obligado a tomar una serie de medidas al respecto. Así, en los meses de noviembre y diciembre del 2008, las autoridades de Montenegro introdujeron diversas modificaciones a la ley bancaria existente, por las cuales el Gobierno garantizó los depósitos bancarios y tomó al mismo tiempo varias medidas económicas anticíclicas que perseguían amortiguar los fuertes efectos de la crisis. Estas medidas anticíclicas se combinaron con otras que perseguían la reducción de los gastos improductivos, al mismo tiempo que se redujeron los impuestos y se aceleró el tiempo de ejecución de los pagos públicos. Estas medidas anticrisis supusieron que el déficit público en el año 2009 se disparara al 9,5 por 100 del PIB. En definitiva, las medidas adoptadas no fueron muy diferentes a las implementadas en otros Estados europeos durante esos años.

Sin embargo, el país retomó la senda del crecimiento a partir del año 2010, cosa que no sucedió en muchas de las economías europeas. A este hecho ayudó enormemente la inversión rusa en inmuebles, que continuó de forma significativa, y es que hay que recordar que en ese año la economía rusa no sufrió tanto la crisis financiera internacional como las economías occidentales. Los precios de las viviendas en el año $\mathbf{2 0 1 0}$ detuvieron la caída notable que experimentaron en el ejercicio anterior, e incluso a finales de año se produjeron ligeros crecimientos en los precios de los inmuebles. También creció ese año el sector industrial, apoyado por el importante desarrollo del sector energético y por el repunte del sector turístico, cuyo gasto aumentó por parte de los ciudadanos extranjeros en un 6,4 por 100 respecto al ejercicio anterior.

También el país avanzó en garantizar los depósitos bancarios, pero a día de hoy dicha cobertura no alcanza a la marcada en la Directiva 2009/14/EC situada en los 100.000 euros y es que el país no cuenta con la capacidad financiera suficiente para acometerla, aunque las autoridades de Montenegro aspiraran a ir incrementando paulatinamente la cobertura de los depósitos bancarios en el futuro.

En definitiva, el Banco Central de Montenegro adoptó mayores estándares de control sobre su sistema bancario, al mismo tiempo que le exigió unos niveles de capitalización superiores a los que se estaban demandando en otros sistemas bancarios occidentales. En el primer trimestre del año 2010, y al igual que habían hecho los bancos centrales de otros países, el Banco Central de Montenegro realizó test de estrés, en los cuales se medía la solvencia de los bancos ${ }^{22}$ ante escenarios hipotéticos de fuertes tensiones económicas y financieras en el país. Los criterios seguidos por las autoridades montenegrinas en su elaboración fueron muy similares a los utilizados por el Banco Central Europeo y la Autoridad Bancaria Europea (EBA). Sin embargo, sus resultados no fueron hechos públicos ya que estos se mantuvieron en la confidencialidad, de acuerdo a lo señalado por la ley que posibilitó su desarrollo. La realización de estos test sirvió para mejorar la confianza de los depositantes en su sistema bancario y, por tanto, contribuyeron a su estabilización, al tiempo que precipitaron la recapitalización de algunas entidades.

La crisis puso de manifiesto las debilidades del sistema bancario montenegrino y esto hizo que las autoridades se mostraran más exigentes en lo que a la capitalización de las entidades se refiere y a exigir a estas un mayor control del riesgo.

\section{Conclusiones}

La crisis del sistema bancario de Montenegro y su resolución no fueron muy diferentes a las acaecidas en otros países europeos, y ello a pesar de las especiales características económicas y políticas del país. La implantación del euro, a pesar $\triangleright$

\footnotetext{
22 Los once bancos integrantes del sistema bancario montenegrino fueron sometidos a los test de stress.
} 
de que sus autoridades no participan en la política monetaria europea de forma activa, ha supuesto, por una parte, una estabilización en sus niveles de precios extraordinaria, al mismo tiempo que ha favorecido la llegada de enormes flujos financieros a su economía. Este hecho disparó la concesión de créditos al sector inmobiliario, como había sucedido en otras economías europeas como la española, irlandesa $o$ islandesa, aunque sus crecimientos fueron mucho más verticales que los de estas y en un periodo de tiempo mucho más reducido, apenas tres años. No obstante, no hay que olvidar que el país contaba con un nivel de infraestructuras e inmobiliario muy poco desarrollado, después de que Montenegro viviera casi 25 años de crisis económica.

Aunque sus flujos de inversión provienen de países de la Unión en su mayor parte, el hecho de que esté situado en el centro de Europa también hace al país receptor de importantes inversiones de otros países vecinos como Rusia o Turquía. Esta mayor diversificación en los flujos de inversiones ha tenido enormes efectos positivos en su economía y, en consecuencia, en su sector bancario.

Montenegro tiene por delante todavía una importante labor de modernización de su legislación bancaria para los próximos años, y ello a pesar de los notables avances que se han producido en los últimos años. La futura adhesión del país a la Unión y su pertenencia a varios organismos internacionales ha tenido una importancia decisiva en lo que a la mejora de su regulación bancaria se refiere, al mismo tiempo que ha favorecido la estabilidad y la confianza en la economía de Montenegro.

\section{Bibliografía}

[1] CENTRAL BANK OF MONTENEGRO (2014). Inflation Report Quarter III, 2014.

[2] CENTRAL BANK OF MONTENEGRO (2015). Bulletin of the Central Bank of February 2015.

[3] CENTRAL BANK OF MONTENEGRO (2010, 2011, 2012). Financial stability report.

[4] INTERNATIONAL MONETARY FUND (2008). «Republic of Montenegro: Financial System Stability Assessment». IMF Country Report $n^{\circ} 8 / 50$.

[5] RADOJE, Z. (2013). «Crisis management and the banking system in Montenegro». Journal of Central Banking Theory and Practice.

[6] RADZIC, J. y YUCE, A (2008). «Banking development in the former Yugoslavian Republics». International Business and Economic Research Journal. vol. 7.

[7] STIBLAR, F. (2009). The impact of the global crisis on Montenegro and west Balkans. Central Bank of Montenegro.

[8] STIBLAR, F. (2010). «The western Balkans in the turmoil of global crisis». Pecob's paper series. University of Ljublijana.

[9] VUCOVIC, S. (2014). «Stress testing of the Montenegrin Banking System with aggregated and bank specific data». Journal of central banking theory and practice.

[10] WORLD BANK (2012). «Montenegro: Preparing for prosperity. Ensuring Sustainability, Connectivity, and Flexibility for Dynamic Growth». Report $n^{\circ}$ 73826-ME. Country Economic Memorandum.

[11] WORLD BANK (2015). Chapter 2: Europe and Central Asia. «Global Economic Prospects» January 2015. 
